\title{
Changes to perceptions of the pros and cons of genetic susceptibility testing after APOE genotyping for Alzheimer disease risk
}

\author{
Kurt D. Christensen, $M P H^{I}$, J. Scott Roberts, PhD ${ }^{I}$, Wendy R. Uhlmann, $M S^{2}$, \\ and Robert C. Green, $M D, M P H^{3}$
}

\begin{abstract}
Purpose: Perceptions about the pros and cons of genetic susceptibility testing are among the best predictors of test utilization. How actual testing changes such perceptions has yet to be examined. Methods: In a clinical trial, first-degree relatives of patients with Alzheimer disease received genetic risk assessments for Alzheimer disease including $A P O E$ disclosure. Participants rated 11 possible benefits associated with genetic testing (pros) and 10 risks or limitations (cons) before genetic risk disclosure and again 12 months afterward. Results: Pros were rated higher than cons at baseline ( 3.53 vs. $1.83, P<0.001)$ and at 12 months after risk disclosure $(3.33$ vs. $1.88, P<0.001)$. Ratings of pros decreased during the 12 -month period ( 3.33 vs. $3.53, P<0.001$ ). Ratings of cons did not change (1.88 vs. $1.83, P=0.199)$ except for a three-item discrimination subscale which increased (2.07 vs. $1.92, P=0.012$ ). Among specific pros and cons, three items related to prevention and treatment changed the most. Conclusion: The process of $A P O E$ genetic risk assessment for Alzheimer disease sensitizes some to its limitations and the risks of discrimination; however, 1-year after disclosure, test recipients still consider the pros to strongly outweigh the cons. Genet Med 2011:13(5):409-414.
\end{abstract}

Key Words: Alzheimer, pros, cons, benefits, discrimination, genetics, risk, APOE, susceptibility testing, education

$T^{\text {tom }}$ he rapid emergence of genetic susceptibility tests for common diseases highlights a need to understand how users appraise them. Perceptions about the benefits and advantages (pros) and the risks and limitations (cons) of genetic susceptibility tests are among the most powerful predictors of test utilization ${ }^{1-4}$ and are central constructs in many popular theories of health behavior and decision making such as the Health Belief Model ${ }^{5}$ and the Theory of Planned Behavior. ${ }^{6}$ Many studies have examined the association between perceived pros and cons and factors such as race, ${ }^{7-9}$ age, ${ }^{9}$ gender, ${ }^{10}$ education, ${ }^{9}$ personal history of disease, ${ }^{11}$ perceived risk of disease, ${ }^{11}$ and medical mistrust. ${ }^{12}$ An important factor that has been neglected in research on such perceptions is the experience of genetic testing itself.

From the ${ }^{1}$ Department of Health Behavior and Health Education, University of Michigan School of Public Health; ${ }^{2}$ Departments of Internal Medicine and Human Genetics, University of Michigan, Ann Arbor, Michigan; and ${ }^{3}$ Departments of Neurology, Medicine (Genetics Program), and Epidemiology, Boston University Schools of Medicine and Public Health, Boston, Massachusetts.

Kurt D. Christensen, MPH, Department of Health Behavior and Health Education, University of Michigan School of Public Health, 1415 Washington Heights, Ann Arbor, MI 48109-2029. E-mail: kdchrist@umich.edu.

Disclosure: The authors declare no conflict of interest.

Submitted for publication August 4, 2010.

Accepted for publication November 18, 2010.

Published online ahead of print January 25, 2011.

DOI: $10.1097 /$ GIM.0b013e3182076bfl
Examining how perceptions of the pros and cons change after actual genetic susceptibility testing should provide insight about how well initial expectations match the real experience. Reasons exist to believe they might not. Misconceptions about genetics and genetic services among the public are widespread. ${ }^{13-17}$ Television shows and movies frequently exaggerate the capabilities of genetic services or focus on highly deterministic forensic uses, ${ }^{18}$ and the media often exaggerate the benefits of genetic research while underrepresenting its risks and limitations. ${ }^{19,20}$ In addition, marketing for consumer genetic services can be confusing or misleading. ${ }^{21,22}$ On the other hand, individuals who seek genetic susceptibility tests tend to be highly educated, ${ }^{23,24}$ satisfaction with decisions about genetic testing is usually high, ${ }^{25,26}$ and evidence to date suggests that many individuals follow through on testing recognizing that it has limitations and risks. ${ }^{27}$ Individuals' understandings about what genetic susceptibility tests can achieve, what they cannot achieve, and the risks they pose should be more realistic after testing than beforehand. However, no study has previously compared pre- and post-test perceptions about the pros and cons of genetic susceptibility testing.

This report explores pre- and post-test perceptions of the pros and cons of genetic susceptibility testing in the context of apolipoprotein E (APOE) testing conducted as part of a genetic risk assessment for Alzheimer disease (AD). The APOE gene has three major alleles: $\epsilon 2, \epsilon 3$, and $\epsilon 4$. The $A P O E \epsilon 4$ allele is among the best predictors of $\mathrm{AD}$ risk, ${ }^{28}$ and $20 \%$ or more of most ethnic groups are carriers of an $\epsilon 4$ allele. ${ }^{29} A P O E$ is considered a susceptibility test because it has limited predictive value: $\epsilon 4$ carriers may live long lives without ever developing the disease and noncarriers still have risk for AD. Although proven prevention strategies are lacking, our previous studies have found that many individuals still perceive genetic susceptibility testing for AD to have substantial utility, from being able to reduce anxiety about $\mathrm{AD}$ risk to informing decisions about long-term care (LTC) insurance. ${ }^{10,30}$ However, these analyses did not explicitly compare pre- and post-test perceptions.

The analysis that follows compares pre- and post-test perceptions about the pros and cons of $A P O E$ genetic susceptibility testing for $\mathrm{AD}$ in the Risk Evaluation and Education for $A L$ zheimer Disease (REVEAL) Study. ${ }^{31}$ On the basis of the research on BRCA1/2 genetic testing decisions showing high post-test satisfaction with decisions, ${ }^{25,26}$ we hypothesized that participants who completed $A P O E$ genetic susceptibility testing for AD would rate its pros higher than its cons. On the basis of the aforementioned possible misconceptions about genetics and genetic services, we also hypothesized that ratings of pros and cons would change after testing. Finally, we tested whether the kind of pretest education that participants received or the results of the genetic risk assessments had any impact on how pretest ratings of the perceived pros and cons changed 12 months after disclosure. 


\section{MATERIALS AND METHODS}

\section{Overview}

Data were analyzed from the second trial of the REVEAL Study, a series of multicenter randomized clinical trials examining the psychosocial and behavioral impact of providing AD susceptibility testing with $A P O E$ genotype disclosure. ${ }^{31}$ In this trial, conducted between 2003 and 2006, participants were randomized into two arms in which they received either a "conventional" or an abbreviated "condensed" pretest educational protocol. The study took place at four academic centers: Boston University School of Medicine in Boston, MA, Case Western Reserve Medical School in Cleveland, OH, Weill School of Medicine in New York, NY, and Howard University School of Medicine in Washington, DC. Preliminary results of the randomized trial have been presented elsewhere ${ }^{32-34}$ and are being reported separately. This article reports a secondary analysis comparing pre- and post-test perceptions of pros and cons.

\section{Participants and procedures}

Participants were adult first-degree relatives (i.e., parents, siblings, or children) of patients with $\mathrm{AD}$, for whom the average age of onset of AD within the family was 60 years or more. Participants completed a baseline assessment that included a telephone interview and self-administered mailed questionnaires and were then randomized to one of two pretest educational protocols. The conventional education arm had in-person educational sessions with a genetic counselor followed by genetic counselor-directed discussions. The condensed education arm substituted educational brochures for the in-person educational sessions and simply provided an opportunity for participants to ask questions. Participants in both arms of the study were provided with their genotype and numerical risk assessments and risk curves based on their APOE genotype, age, gender, self-identified ethnicity, and family history. ${ }^{35,36}$ Scripted messages and printed materials in both arms addressed the same topics, including pros and cons, as shown in Table 1. Informed consent documents signed at enrollment and again just before the blood draw for genotyping reiterated the same points.

After blood draw and genotyping, participants in both arms received personalized $\mathrm{AD}$ risk estimates through age 85 years ranging from $13 \%$ to $77 \%$ depending on gender, self-identified ethnicity, family history of $\mathrm{AD}$, and $A P O E$ genotype. Participants also received remaining $\mathrm{AD}$ risk estimates that incorporated their current ages. Participants were then followed up for a year after disclosure.

Protocols were approved by an External Advisory Board and institutional review boards at each study site.

\section{Measures \\ Demographics}

Gender, ethnicity, age, education, employment status, and income data were collected via self-report during the initial phone interview.

\section{Educational protocol}

Subjects were coded as receiving the conventional or condensed educational protocols based on randomization status.

\section{APOE genotype}

$A P O E$ genotype was dichotomized depending on whether a participant carried at least one copy of the $A P O E \in 4$ allele associated with increased risk for AD or not.

\section{Pros and cons}

Perceived pros and cons of genetic susceptibility testing for $\mathrm{AD}$ were assessed in the baseline written questionnaire administered before pretest education and again in the 12-month follow-up questionnaire. Scales measuring perceived pros and cons of genetic testing for AD were modified from research on genetic testing for hereditary breast and ovarian cancer ${ }^{4,37}$ and implemented in subsequent studies of attitudes about AD susceptibility testing, including prior REVEAL Study trials..$^{3,10}$ Questionnaires included a section that offered reasons "why someone might take a genetic test for AD" (Pros) and a separate section that offered reasons "why someone might not want to take a genetic test for AD" (Cons). See Table 3 for a list of the specific items. Participants rated how important each reason was to them on a Likert scale of 1 (not at all) to 5 (extremely). Overall Pros (range: 1-5) was calculated by taking the mean of the ratings of the 11 pros items, with higher scores indicating more favorable perceptions about testing. Overall Cons (range: 1-5) was similarly calculated taking the mean of the ratings of the 10 cons items, with higher scores indicating more reserva-

Table 1 Pros and cons of AD genetic risk assessment as described in educational materials and informed consent forms

Pros Cons

It may encourage you to stay abreast of new developments in $\mathrm{AD}$ treatment and prevention.

It may motivate you to engage in activities that may help prevent or delay the onset of AD.

It may satisfy your curiosity about your chances of developing the disease.

You may use your risk assessment to help make long-term decisions.

Receiving a lower risk estimate may reduce your anxiety about developing the disease.
There are no proven ways to prevent AD from developing.

Your risk profile is only an interpretation based on our current knowledge and will not give you a simple "yes" or "no" answer, nor will it indicate at what age AD may develop.

Employers or insurance companies could ask you about your risk information and use it to deny coverage or change your policy rates.

If you tell others about your results, there is no guarantee that your results will remain confidential.

You may find it harder to cope with your concerns about AD after having your risk estimate.

It is possible that you or a loved one may misinterpret the results, causing undue stress or false reassurance about your chances of developing AD. 
tions about testing. Confirmatory factor analysis showed strong internal consistency for both scales: Cronbach's alpha was 0.81 at baseline and 0.87 at 12 months for the pros scale and 0.81 for the cons scale at both baseline and 12 months.

Exploratory factor analysis suggested that three items from the cons scale, namely "the results could affect my employment," "the results could affect my health insurance," and "the results could change how people look or act toward me," comprised a Discrimination Fears subscale. In addition to face validity, the subscale demonstrated acceptable internal consistency, with Cronbach's alpha scoring 0.70 at baseline and 0.79 at 12 months. We therefore conducted additional analyses on the mean ratings of items in this subscale (range: 1-5).

Nonresponse on items specific to children (up to 20 instances on each item) was scored "not at all important" if the subject reported having no children. Nonresponse on the item, "the results could affect my employment" (13 instances) was also scored "not at all important" if the subject reported being retired. Nonresponses on all other items (up to six instances per item) were imputed using the median scores across subjects on the items.

\section{Data analysis}

Only participants who completed the 12-month follow-up were analyzed. Chi-square analyses, Pearson product-moment correlations, and Spearman rank correlations were used to test whether demographic or study-related factors were associated with study dropout between the baseline assessment and the 12-month follow-up. Paired $t$-tests were used to compare overall pros against overall cons. Paired $t$-tests were also used to examine how overall pros, overall cons, and discrimination fears changed between baseline and follow-up, and independent samples $t$-tests were used to examine whether educational protocols or $A P O E$ genotype were associated with such changes. Finally, paired $t$-tests were used to assess whether individual reasons for and against testing changed between baseline and follow-up, and independent samples $t$-tests were used to determine whether changes from baseline differed according to educational protocol or $A P O E$ genotype. One-sided tests were used to test the hypothesis that overall pros would be rated higher than overall cons. All other tests were two-sided.

\section{RESULTS}

\section{Demographics and study retention}

Three hundred and twenty-nine participants completed the baseline survey, and 299 of them (91\%) received AD risk estimates with APOE genotype disclosure. Ultimately, 293 participants $(89 \%)$ retained through the 12-month follow-up and were included in data analyses. Demographics of these participants are presented in Table 2. A majority of participants referred themselves to the study, learning about the study through presentations given by investigators from the REVEAL Study, other research studies, postings on the internet, community newsletters, health fairs, or via the Alzheimer's Association.

Three demographic factors were found to be associated with study dropout between the baseline assessment and the 12month follow-up: no current employment $\left(\chi^{2}=8.64, P=\right.$ $0.003)$, lower household income $(\rho=0.21, P<0.001)$, and less education $(r=0.18, P=0.001)$. Higher ratings of overall pros at baseline were associated with study dropout before the 12month follow-up $(r=0.11, P=0.049)$ but not before results disclosure $(r=0.01, P=0.920)$. Higher ratings of overall cons
Table 2 Participant demographics $(n=273)$ at the 12-month follow-up

Median age (range) $57(33-86)$

Percent of female $70 \%$

Mean years of education (range)

Full-time or part-time employed

Median income bracket

$\$ 70 \mathrm{~K}-\$ 99 \mathrm{~K}$

Race: Percent of African American

$19 \%$

Educational protocol

Condensed

$67 \%$

Traditional

$33 \%$

Has $A P O E \in 4$ allele

$41 \%$

Study site

Boston University

$31 \%$

Weill School of Medicine

$29 \%$

Case Western Reserve

$22 \%$

Howard University

$18 \%$

at baseline were associated with study dropout by disclosure ( $r=0.17, P=0.003)$ and the 12-month follow-up ( $r=0.16$, $P=0.004)$. Discrimination fears were not associated with study dropout by 12 months ( $r=0.08, P=0.172)$

\section{Overall pros, overall cons, and discrimination fears}

Participants rated the items on the pros scale much higher than items on the cons scale both before and after testing as hypothesized. At baseline, the mean overall pros score was 3.53, whereas the mean overall cons score was $1.83(t=33.4$, $P<0.001)$. At 12 months, the mean overall pros score was 3.33 , whereas the mean overall cons score was $1.88(t=26.2$, $P<0.001)$. Changes from baseline were also noted. On average, overall pros were rated slightly lower 12 months after disclosure than at baseline (3.33 vs. $3.53, t=4.82, P<0.001$ ). Overall cons were rated approximately the same at both time points (1.88 vs. $1.83, t=1.29, P=0.199)$ but scores on the discrimination fears scale were higher at 12 months than baseline (2.07 vs. $1.92, t=2.54, P=0.012)$. Changes on all scales were not associated with the educational protocol participants received (pros: $\Delta=-0.01, t=-0.07, P=0.944$; cons: $\Delta=$ $0.02, t=0.29, P=0.769$; and discrimination fears: $\Delta=0.01$, $t=0.06, P=0.954$ ) or $A P O E$ genotype (overall pros: $\Delta=$ $-0.06, t=-0.67, P=0.502$; overall cons: $\Delta=0.02, t=0.22$, $P=0.830$; and discrimination fears: $\Delta=0.08, t=0.65, P=$ $0.517)$.

\section{Changes to individual pros and cons}

Table 3 summarizes how ratings of individual pros and cons changed at follow-up. Six pros were rated lower at 12 months compared with baseline. The greatest change occurred on the item "to seek information on preventive measures," where $43 \%$ of participants rated the benefit lower at follow-up than at baseline. Changes on that item were associated with $A P O E$ genotype, where $\epsilon 4$ carriers lowered their ratings less at follow-up than noncarriers $(\Delta=0.28, t=2.00, P=0.047)$. "To give information about my children's possible risk of AD" was 
Table 3 Ratings of individual pros and individual cons at baseline and at 12 months ordered by magnitude of change $(1=$ not at all important, $5=$ extremely important $)$

\begin{tabular}{|c|c|c|c|c|}
\hline & $\begin{array}{l}\text { Mean at } \\
\text { baseline }\end{array}$ & $\begin{array}{l}\text { Mean at } \\
12 \text { months }\end{array}$ & $\Delta$ & $P$ \\
\hline \multicolumn{5}{|l|}{ Pros } \\
\hline To seek information on preventative measures & 4.26 & 3.75 & -0.51 & $<0.001$ \\
\hline The need to make arrangements for my long-term care & 3.67 & 3.31 & -0.36 & $<0.001$ \\
\hline To know more about my risk in case better treatments become available & 4.26 & 3.91 & -0.35 & $<0.001$ \\
\hline The desire to contribute to research on $\mathrm{AD}$ & 4.11 & 3.86 & -0.25 & $<0.001$ \\
\hline The desire to start doing things sooner than I had planned to & 3.37 & 3.18 & -0.19 & 0.018 \\
\hline To give information about my children's possible risk of $\mathrm{AD}$ & 3.01 & 2.82 & -0.19 & 0.020 \\
\hline The need to arrange my personal affairs & 3.69 & 3.56 & -0.13 & 0.097 \\
\hline To confirm the feeling that I might already be developing AD & 2.32 & 2.19 & -0.13 & 0.099 \\
\hline To put my mind at ease if I found out I was not at risk for $A D$ & 3.53 & 3.45 & -0.08 & 0.346 \\
\hline The need to prepare my family for my possible illness & 3.43 & 3.38 & -0.05 & 0.513 \\
\hline Curiosity & 3.17 & 3.26 & .09 & 0.256 \\
\hline \multicolumn{5}{|l|}{ Cons } \\
\hline There is no way to cure or prevent $\mathrm{AD}$ & 1.93 & 2.18 & .25 & 0.007 \\
\hline $\begin{array}{l}\text { The test does not give me a definite answer about whether I might get } \\
\text { AD or not }\end{array}$ & 2.13 & 2.30 & .17 & 0.017 \\
\hline It could make me worry about my children's risk of getting $\mathrm{AD}$ & 1.81 & 1.79 & -0.02 & 0.727 \\
\hline My family does not think it is a good idea for me & 1.25 & 1.20 & -0.05 & 0.350 \\
\hline It would be too upsetting to find out I'm at risk for $\mathrm{AD}$ & 1.96 & 1.88 & -0.08 & 0.289 \\
\hline The test results might upset my loved ones & 2.10 & 1.97 & -0.13 & 0.075 \\
\hline The test procedure would be too burdensome & 1.37 & 1.24 & -0.13 & 0.011 \\
\hline \multicolumn{5}{|l|}{ Discrimination fears } \\
\hline The results could affect my employment & 1.60 & 1.85 & 0.25 & 0.001 \\
\hline The results could affect my health insurance & 2.37 & 2.48 & 0.11 & 0.184 \\
\hline The results could change how people look at or act toward me & 1.78 & 1.88 & 0.10 & 0.153 \\
\hline
\end{tabular}

also rated lower at follow-up, but $\epsilon 4$ carriers lowered their ratings more at follow-up than noncarriers $(\Delta=-0.47, t=$ $2.97, P=0.003)$. Other individual items rated lower at follow-up than baseline, regardless of genotype, were "the need to make arrangements for my long-term care," "to know more about my risk in case better treatments become available," "the desire to contribute to research on $\mathrm{AD}$," and "the desire to start doing things sooner than I had planned to." Changes in ratings of individual pros items were not associated with $A P O E$ genotype or educational protocol except for the two items noted above.

The ratings of four con items also showed changes. Three that were rated higher at follow-up than baseline were "the test does not give me a definite answer about whether or not I might get $\mathrm{AD}$," "there is no way to cure or prevent $\mathrm{AD}$," and "the results could affect my employment." In contrast, "the test procedure would be too burdensome" was rated lower at follow-up than at baseline. No changes were associated with $A P O E$ genotype or educational protocol.

\section{DISCUSSION}

This is the first analysis to explore how perceptions about the pros and cons about genetic susceptibility tests change after actual testing. Our data supported the hypothesis that people who seek and obtain $A P O E$ genetic testing as part of obtaining $\mathrm{AD}$ risk assessment retain strong positive attitudes about the experience but generally reduce their endorsements of the pros and increase their concerns about risk of discrimination after undergoing testing. Among the topics where perceptions of the pros were diminished after disclosure, this drop was particularly striking in the items "to seek information about preventative measures" and "to know about risk in case better treatments become available." Similarly, there was an increase in the perceived con item "lack of a cure or prevention." One of the main reasons $A P O E$ testing of asymptomatic patients for $\mathrm{AD}$ risk is not part of standard clinical practice is the absence of options to prevent or delay disease onset, ${ }^{38-40}$ and educational materials in the REVEAL Study emphasized that "there are no 
proven ways to prevent Alzheimer disease." Despite this clear disclaimer, it is possible that some participants sought susceptibility testing thinking that genetic susceptibility testing would expose them to new strategies for reducing or delaying AD risk, especially when conducted as part of research. Anecdotally, many participants asked REVEAL Study personnel whether the investigative team was also associated with prevention and treatment trials, although such queries were not formally tracked.

At the same time, $A P O E \in 4$ carriers did not lower their rating of "to seek information on preventative measures" as much as noncarriers did, suggesting that they found greater utility to the genetic risk assessment in terms of thinking about prevention measures. This finding is consistent with prior analyses of REVEAL Study data showing $\epsilon 4$ carriers to be more likely to report health behavior changes than noncarriers, particularly in their consumption of dietary supplements and vitamins. ${ }^{41,42}$ It seems that those who learn they are $\epsilon 4$ carriers may feel justified in using unproven preventative measures. If so, clinicians who provide predictive genetic testing should be sure that efficacy expectations about such measures are realistic and that test recipients do not pursue strategies - such as off-label use of pharmaceuticals - that may be detrimental to their health.

Another theme that emerged in the data related to the risk of future disability. "To make arrangements for long-term care" and "to start doing things sooner" were reasons for testing that were, on average, rated lower after testing than before. This finding was surprising given prior REVEAL Study data showing that $A P O E$ genetic susceptibility test results can inform the purchase of LTC insurance. ${ }^{30,43}$ It is possible that participants who hoped to use testing to inform decisions about the future, such as LTC insurance purchasing, were dissuaded because of greater sensitivity to the limited positive and negative predictive value of $A P O E$ for AD. Supporting this argument, participants rated the lack of a definitive answer about future $\mathrm{AD}$ onset as a stronger con at follow-up.

Participants also tended to rate the reason "to give information about my children's possible risk for AD" lower at follow-up than at baseline. The change varied by $A P O E$ status, with $\epsilon 4$ carriers lowering their ratings more than noncarriers. This difference may be attributable to concern or guilt about passing increased risk for AD risk along to children, supported by evidence that carriers feel more test-specific distress in the short-term than noncarriers. ${ }^{31}$ Another possibility is that carriers may consider sharing "bad" news to be more stressful than sharing "good" news.

A final benefit that was rated lower after testing was "the desire to contribute to research on AD." This finding is interesting given prior findings that endorsement of this reason predicted follow-through on intentions to seek testing in prior analyses. ${ }^{10}$ It is possible that the burdens of study participation-which included regular follow-ups and completion of questionnaires over a 1-year period after disclosure-tempered the altruism many participants felt when they originally enrolled in the study, and anecdotal evidence suggested that some participants thought the surveys and time required for the study were excessive. On the other hand, participants tended to rate testing burdens lower at follow-up than at baseline. It may simply be that testing was interesting while study follow-up was less so.

The increases in concern about discrimination merit attention considering federal legislation enacted after data collection ended in 2006. The Genetic Information Nondiscrimination Act of 2008 has been hailed as a success for genomic medicine because it provides federal protections against employment and health insurance discrimination based on genetic information, including genetic test results. ${ }^{44}$ Although many such protections had already been provided in a patchwork of state legislationsincluding all states and districts hosting REVEAL Study sites at the time of the study - the passage of Genetic Information Nondiscrimination Act has likely raised awareness about the existence of legal protections, especially considering the media attention its passage has generated after 13 years of debate. Discrimination fears related to genetic testing may not be so strong in the future.

Several limitations should be kept in mind while interpreting results. Our methodology does not allow us to make determinations about what reasons may have caused the changes in perceptions of pros and cons. After participants completed the baseline assessment, participants received educational preparation for the study, underwent a second informed consent step, and had in-person discussions with a clinician. In each of those steps, participants received information about the benefits, risks, and limitations of $\mathrm{AD}$ genetic susceptibility testing, and any or all of these activities could have contributed to changes in perceptions of pros and cons. Furthermore, participants lacking data at 12 months were omitted from analysis, possibly biasing results. Among this group were those with lower presumed socioeconomic status (those with the less education, lower household incomes, or were not currently employed) in addition to those who perceived greater pros and greater cons at baseline. In addition, our close-ended survey items are unlikely to have captured all pros and cons relevant to a given participant. Finally, significance levels were not adjusted to account for the number of comparisons conducted because the study was not powered for this secondary analysis, thus increasing the risk for false-positive findings.

Nevertheless, the findings from this analysis have important implications for the way genetic services are delivered. Our analyses show strong positive attitudes toward genetic susceptibility testing for $\mathrm{AD}$, even after a genetic risk assessment that includes $A P O E$ genotyping. Additional genetic tests associated with risk for $\mathrm{AD}$ and tests for other untreatable diseases are likely to become available in the future. ${ }^{24}$ If so, we can expect those who have already undergone AD susceptibility testing to have favorable attitudes toward the new ones.

Finally, if post-test ratings represent a more accurate appraisal of the benefits, risks, and limitations of $A P O E$ genetic testing for $\mathrm{AD}$ risk, then the minor changes observed in our analysis demonstrate slight discordance between how participants anticipated they would use genetic risk information and how they actually used it. Experts tend to agree that the best decisions are made when expectations are brought into alignment with true outcomes through a process of education and deliberation. ${ }^{45}$ The condensed educational protocol tested in the second REVEAL Study trial imposed substantially less burdens on both clinicians and test recipients (i.e., one fewer study visit and a reduction in clinic time of over 40 minutes). The lack of differences in perceptions of cons and pros by educational protocol in our study suggests that condensed protocols can have the same effect as more time-consuming ones, at least on altering the perceptions individuals have about what $A P O E$ testing can achieve. As genetic testing for susceptibility becomes more common, providers will need to seek the right balance between feasibility and effectiveness in pretest education to ensure that people seeking such testing are making informed decisions. 


\section{ACKNOWLEDGMENTS}

This study was funded by the following NIH grants: HG02213, HG005092, AG027841, AG09029, AG13846, RR00533, and RR10284.

The authors thank Erin Linnenbringer, Sarah Tersegno, and Lindsay Zausmer at the University of Michigan School of Public Health for their assistance with manuscript preparation.

\section{REFERENCES}

1. Kasparian NA, Meiser B, Butow PN, Simpson JM, Mann GJ. Genetic testing for melanoma risk: a prospective cohort study of uptake and outcomes among Australian families. Genet Med 2009;11:265-278.

2. Roberts JS, Connell CM, Cisewski D, Hipps YG, Demissie S, Green RC. Differences between African Americans and whites in their perceptions of Alzheimer disease. Alzheimer Dis Assoc Disord 2003;17:19-26.

3. Roberts JS. Anticipating response to predictive genetic testing for Alzheimer's disease: a survey of first-degree relatives. Gerontologist 2000; 40:43-52.

4. Lerman C, Narod S, Schulman K, et al. BRCA1 testing in families with hereditary breast-ovarian cancer. A prospective study of patient decision making and outcomes. JAMA 1996;275:1885-1892.

5. Rosenstock IM. Historical origins of the Health Belief Model. Health Educ Monogr 1974;2:328-335.

6. Ajzen I. Attitudes, personality, and behavior. Homewood: Dorsey Press, 1988.

7. Hughes C, Gomez-Caminero A, Benkendorf J, et al. Ethnic differences in knowledge and attitudes about BRCA1 testing in women at increased risk. Patient Educ Couns 1997;32:51-62.

8. Hipps YG, Roberts JS, Farrer LA, Green RC. Differences between African Americans and Whites in their attitudes toward genetic testing for Alzheimer's disease. Genet Test 2003;7:39-44.

9. Donovan KA, Tucker DC. Knowledge about genetic risk for breast cancer and perceptions of genetic testing in a sociodemographically diverse sample. J Behav Med 2000;23:15-36.

10. Roberts JS, LaRusse SA, Katzen H, et al. Reasons for seeking genetic susceptibility testing among first-degree relatives of people with Alzheimer disease. Alzheimer Dis Assoc Disord 2003;17:86-93.

11. Kessler L, Collier A, Brewster K, et al. Attitudes about genetic testing and genetic testing intentions in African American women at increased risk for hereditary breast cancer. Genet Med 2005;7:230-238.

12. Thompson HS, Valdimarsdottir HB, Jandorf L, Redd W. Perceived disadvantages and concerns about abuses of genetic testing for cancer risk: differences across African American, Latina and Caucasian women. Patient Educ Couns 2003;51:217-227.

13. Genetics and Public Policy Center. Chapter 2: Public awareness and knowledge. In: Kalfoglou A, Suthers K, Scott J, Hudson K, eds. Reproductive genetic testing: what America thinks. Washington, DC: Genetics and Public Policy Center, 2004:7-10.

14. National Science Board. Science and technology: public attitudes and understanding. In: Science and engineering indicators 2008, Vol 1. Arlington: National Science Foundation, 2008:7-1:7-47.

15. Lanie AD, Jayaratne TE, Sheldon JP, et al. Exploring the public understanding of basic genetic concepts. J Genet Couns 2004;13:305-320.

16. Christensen KD, Jayaratne TE, Roberts JS, Kardia SLR, Petty EM. Understandings of basic genetics in the United States: results from a national survey of black and white men and women. Public Health Genomics 2010;13:467-476.

17. Singer E, Antonucci T, Van Hoewyk J. Racial and ethnic variations in knowledge and attitudes about genetic testing. Genet Test 2004;8:31-43.

18. Nelkin D, Lindee MS. The DNA mystique: the gene as a cultural icon. Ann Arbor: University of Michigan, 2004.

19. Bubela TM, Caulfield TA. Do the print media "hype" genetic research? A comparison of newspaper stories and peer-reviewed research papers. CMAJ 2004; 170:1399-1407.

20. Holtzman NA, Bernhardt BA, Mountcastle-Shah E, Rodgers JE, Tambor E, Geller G. The quality of media reports on discoveries related to human genetic diseases. Community Genet 2005;8:133-144.
21. Matloff E, Caplan A. Direct to confusion: lessons learned from marketing BRCA testing. Am J Bioeth 2008;8:5-8.

22. Gray S, Olopade OI. Direct-to-consumer marketing of genetic tests for cancer: buyer beware. J Clin Oncol 2003;21:3191-3193.

23. Meiser B, Dunn S. Psychological effect of genetic testing for Huntington's disease: an update of the literature. West J Med 2001;174:336-340.

24. Roberts JS, Barber M, Brown TM, et al. Who seeks genetic susceptibility testing for Alzheimer's disease? Findings from a multisite, randomized clinical trial. Genet Med 2004;6:197-203.

25. Bluman LG, Rimer BK, Sterba KR, et al. Attitudes, knowledge, risk perceptions and decision-making among women with breast and/or ovarian cancer considering testing for BRCA1 and BRCA2 and their spouses. Psychooncology 2003;12:410-427.

26. Klemp JR, O'Dea A, Chamberlain C, Fabian CJ. Patient satisfaction of BRCA1/2 genetic testing by women at high risk for breast cancer participating in a prevention trial. Fam Cancer 2005;4:279-284.

27. Bloss CS, Ornowski L, Silver E, et al. Consumer perceptions of direct-toconsumer personalized genomic risk assessments. Genet Med 2010;12:556566.

28. Morris JC. Dementia update 2005. Alzheimer Dis Assoc Disord 2005;19: $100-117$.

29. Corbo RM, Scacchi R. Apolipoprotein E (APOE) allele distribution in the world. Is APOE*4 a 'thrifty' allele? Ann Hum Genet 1999;63:301-310.

30. Zick CD, Mathews CJ, Roberts JS, Cook-Deegan R, Pokorski RJ, Green RC. Genetic testing for Alzheimer's disease and its impact on insurance purchasing behavior. Health Aff 2005;24:483-490.

31. Green RC, Roberts JS, Cupples LA, et al. A randomized trial of APOE genotype disclosure for risk of Alzheimer's disease: the REVEAL Study. $N$ Engl J Med 2009;361:245-254

32. Green RC, Roberts JS, Linnenbringer E, et al. Impact of a condensed protocol for disclosing genetic susceptibility for Alzheimer's disease: the REVEAL Study. Neurology 2006;66:A349-A350.

33. Roberts $\mathrm{S}$, Linnenbringer $\mathrm{E}$, Butson $\mathrm{MB}$, et al. Impact of a condensed protocol for disclosing APOE genotype to first-degree relatives of people with Alzheimer's disease. Alzheimers Dement 2008;4(4, suppl 1):T454$\mathrm{T} 455$.

34. Green RC, Roberts JS, Chen C, et al. Comparing the impact of a condensed vs extended protocol for disclosure of APOE to relatives of patients with AD: the REVEAL Study. Alzheimers Dement 2007;3(3, suppl 1):S184.

35. Cupples LA, Farrer LA, Sadovnick AD, Relkin N, Whitehouse P, Green RC. Estimating risk curves for first-degree relatives of patients with Alzheimer's disease: the REVEAL Study. Genet Med 2004;6:192-196.

36. Christensen KD, Roberts JS, Royal CDM, et al. Incorporating ethnicity into genetic risk assessment for Alzheimer disease: the REVEAL Study experience. Genet Med 2008;10:207-214.

37. Lerman C, Seay J, Balshem A, Audrain J. Interest in genetic testing among first-degree relatives of breast cancer patients. Am J Med Genet 1995;57: 385-392.

38. Consensus statement on predictive testing for Alzheimer disease. Alzheimer Dis Assoc Disord 1995;9:182-187.

39. Apolipoprotein E genotyping in Alzheimer's disease. National Institute on Aging/Alzheimer's Association Working Group. Lancet 1996;347:10911095.

40. American College of Medical Genetics (ACMG)/American Society of Hum Genet (ASHG) Working Group on ApoE and Alzheimer disease. Statement on use of apolipoprotein E testing for Alzheimer disease. JAMA 1995;274: 1627-1629.

41. Chao S, Roberts JS, Marteau TM, Silliman R, Cupples LA, Green RC. Health behavior changes after genetic risk assessment for Alzheimer disease: the REVEAL Study. Alzheimer Dis Assoc Disord 2008;22:94-97.

42. Vernarelli JA, Roberts JS, Hiraki S, Chen CA, Cupples LA, Green RC. Effect of Alzheimer disease genetic risk disclosure on dietary supplement use. Am J Clin Nutr 2010;91:1402-1407.

43. Taylor DH Jr, Cook-Deegan RM, Hiraki S, Roberts JS, Blazer DG, Green RC. Genetic testing for Alzheimer's and long-term care insurance. Health Aff 2010;29:102-108.

44. Hudson KL, Holohan MK, Collins FS. Keeping pace with the times-the Genetic Information Nondiscrimination Act of 2008. N Engl J Med 2008; 358:2661-2663.

45. Elwyn G, Miron-Shatz T. Deliberation before determination: the definition and evaluation of good decision making. Health Expect 2010;13:139-147. 